\title{
A Milestone in Codifying the Wisdom of Traditional Oriental Medicine: TCM, Kampo, TKM, TVM-WHO International Standard Terminologies on Traditional Medicine in the Western Pacific Region
}

\author{
Seung-Hoon Choi ${ }^{1}$ and II-Moo Chang $^{2}$ \\ ${ }^{1}$ College of Oriental Medicine, Kyung Hee University, Seoul 130-701 and ${ }^{2}$ Natural Products Research Institute, \\ Seoul National University, Seoul 151-742, Korea
}

\begin{abstract}
The WHO published a dictionary-type book entitled 'WHO International Standard Terminologies on Traditional Medicine in the Western Pacific Region' which has a total of 3259 technical terms which have been commonly used in traditional Chinese (TCM), Japanese (Kampo), Korean (TKM) and Vietnamese (TVM) medicines. In this comprehensive guide, each term has the English expression, the original Chinese character and a concise English definition. The book covers 3106 terms from basic theories, diagnostics, diseases, various therapeutics including acupuncture and moxibustion and even the English wording of 153 titles which are considered the most important traditional medical classics published in these four countries. A prominent feature of the compilation is the codification format that assigns numbers in hundred decimal units for each category of the section. This type of coding system provides the flexibility for adding more terminologies in the future and is useful for constructing a database for the retrieval of various published scientific articles. Overall, the usage of these standard terminologies is highly desirable to deliver accurate meanings, and ultimately to avoid a variety of expressions for a single term in different scientific manuscripts on Oriental medicine.
\end{abstract}

Keywords: Oriental medicine - standard terminology - traditional medicine - WHO

\section{Overview: role of WHO}

In mid-2007, the WHO Western Pacific Regional Office (WHO WPRO) laid the first cornerstone by constructing a link between the modern and traditional medicines through publishing a dictionary-type book entitled 'WHO International Standard Terminologies on Traditional Medicine (TM) in the Western Pacific Region (WHO ISTT).' As the Forward and the Acknowledgement sections of the book indicate, this valuable book is the first outcome within the standardization framework using an evidence-based approach under the traditional

For reprints and all correspondence: Il-Moo Chang, Natural Products Research Institute, Seoul National University, Seoul 151-742, Korea.

Tel: (+82-2) 880 2478; Fax: (+82-2) 745 1015;

E-mail: changim@snu.ac.kr medicine program of the WHO WPRO. In addition, a series of books on standard acupuncture point location and standard disease classification follow it.

The WHO ISTT was born from four years' hard work in collaboration with a number of experts. There were three consultation meetings from 2004 to 2005, and an additional 2 years of editorial work, including the printing, from 2005 to 2007. At the consultations meetings, the expert working group showed its commitment through long discussions and debates, sometimes until the early hours of the morning, to craft an accurate definition for each term. Taking a glimpse at the contents of book, a total of 3259 technical terminologies have been compiled from the commonly used in traditional Chinese (TCM), Japanese (Kampo), Korean (TKM) and Vietnamese medicine (TVM). Also, each term is 
Table 1. Coding System of TOM



expressed in English, the original Chinese character and its definition is concisely expressed in English. The book covers 3106 terms from basic theories, diagnostics, diseases, various therapeutics including acupuncture and moxibustion and even the English wordings of a total of 153 titles of important traditional medical classics published in those four countries.

As mentioned in the book, the total number of terms, just over 3000, apparently covers only the basic nomenclature used in Traditional Oriental Medicine (TOM), and these are seen as the basis, the fundamental information, for a better understanding in terms of education, training, practice and research. However, the book seems to have some limitations, and needs further development in the standard expression related to the formula titles of herbal medicinal prescriptions in English. It has been recorded that approximately 100000 formula titles are expressed by the Chinese alphabet (1). Until now, the same formula title has been expressed differently in English in different countries according to the country-specific phonetic rules. For instance, a famous tonic, a multi-herb formula which consists of 10 herbal components, is expressed in English as Juzen Taiho To by Japanese, Shi Quan Da Bu Tang by Chinese and Sib Jeon Dae Bo Tang by Koreans. A number of scientific articles containing this formula have been reported, and listed separately in the PubMed retrieval system. Western scientists who do not have a basic knowledge in the Chinese alphabet would probably consider those formula titles as different herbal prescriptions. Therefore, I think the next step that the WHO ISTT needs to take is further investment to facilitate standard nomenclature of herbal formula titles. Nonetheless, the WHO ISTT is certainly fulfilling the role of providing basic information to understand the fundamental knowledge of TOM.

\section{Characteristics of codification system of TOM}

A characteristic feature of the editorial format for the compilation is to assign the codes with numbers in hundred decimal units for each category of the section. For instance, the General Section is coded with 0.0.0, and 0.0.1. for Traditional Medicine, and 0.0.2. and 0.0.3. for Oriental Medicine. For example, for the Basic Theories Section, 1.0.0. is assigned, and is followed by 1.1.0 for Essential Qi Theory, Yin-Yang Theory and Five Phase Theory. This coding system based on numbers provides two advantages: first, it becomes easy to identify each term, and second, it is convenient for adding more terms when the book is revised in the future. Additionally, this flexible type of coding system will be very useful in constructing the database when the total number of terminologies is increased in the future.

The distribution patterns of terminologies are summarized in Table 1 in accordance with each section of the 
contents. Looking at it, we can fully appreciate the reality and present status of Oriental Medicine. For instance, there are diverse and unique terms in theories which are based essentially on the concept of Oriental cosmology. There are also a variety of diagnostics to identify the diseases even without using modern and advanced tools, implying a prerequisite of much experience for doctors to make the accurate diagnosis. In addition, it is interesting that some areas such as the gynecology-obstetrics, pediatrics, ophthalmology, otorhinolaryngo-stomatology and orthopedics-traumatology were branched from an early time, and they are available even now at many TOM hospitals.

\section{Conclusion: implications for TOM}

The WHO has made great efforts in utilizing the various traditional medicines from all over the world under the motto of 'health for all'. This book is witness to this philosophy, and the accomplishment of this book will be a basis for the further progress in standardizing the various areas of the traditional medical sciences. The standardization of acupuncture point locations was already made, and the standard disease classification in terms of traditional medicine will be soon released. Moreover, the Guide of Clinical Practice Guidelines has been studied in order to harmonize it with the International Conference on Harmonization of Technical
Requirements for Registration of Pharmaceuticals for Human Use (ICH); Good Clinical Practice (GCP) guidelines (2). On another front, international collaboration with the WHO TM team has been also begun to develop a possible English coding system to express the titles of herbal formulae, which has over 100000 titles and is described in Chinese characters, in relation with the Anatomy-Therapeutic Code (ATC) of drugs (2).

Information related to the WHO International Standard Terminologies on Traditional Medicine in the Western Pacific Region is as follows.

(i) A publication of the WHO in 2007: hard- and soft-covered versions (349 pages).

(ii) ISBN 9789290612483 (NLM classification: WB50).

(iii) Whole contents can be seen and possibly downloaded from the WHO Western Pacific Region's website (www.wpro.who.int).

\section{References}

1. Yi YD, Chang IM. An overview of traditional Chinese herbal formulae and a proposal of a new code system for expressing the formula titles. eCAM 2004:1:125-32.

2. Chang IM. Initiative for developing evidence-based standardization of traditional Chinese medical therapy in the Western Pacific Region of the World Health Organization. eCAM 2004;1:337-41.

Received October 10, 2008; accepted December 5, 2008 


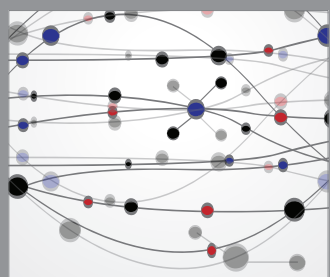

The Scientific World Journal




Gastroenterology

Research and Practice
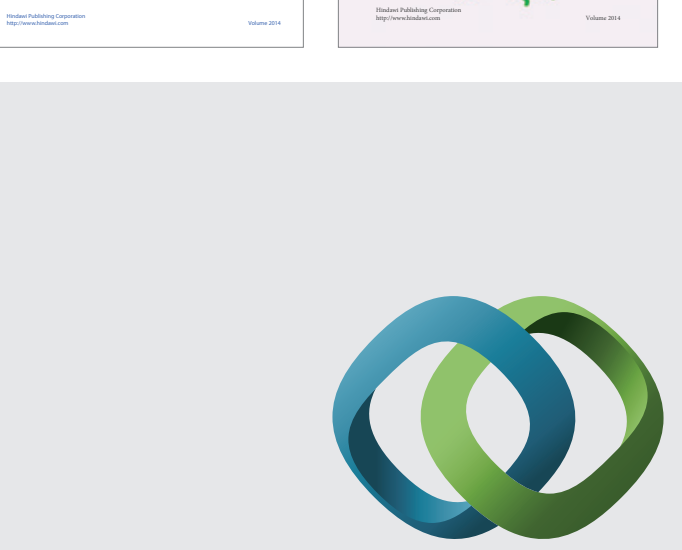

\section{Hindawi}

Submit your manuscripts at

http://www.hindawi.com


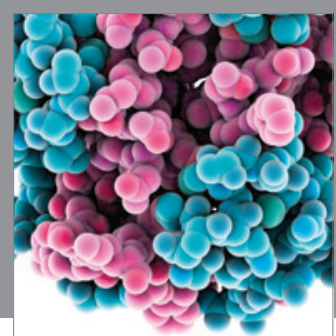

Journal of
Diabetes Research

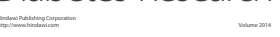

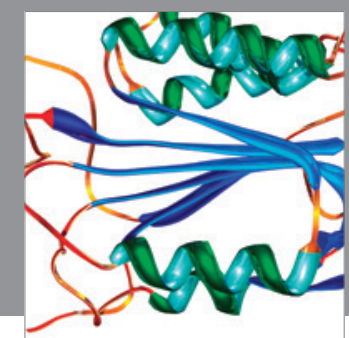

Disease Markers
